\title{
Substance Adverse Event
}

National Cancer Institute

\section{Source}

National Cancer Institute. Substance Adverse Event. NCI Thesaurus. Code C101243.

An unexpected or dangerous reaction to a substance (e.g., food, environmental ag ent).

(ACC) 